\title{
Erratum to: Sensitivity analysis of continuous-time models for ecological and evolutionary theories
}

Romain Richard $^{1,3} \cdot$ Jérôme Casas $^{2} \cdot$ Edward McCauley $^{1}$

Published online: 30 October 2015

(C) Springer Science+Business Media Dordrecht 2015

Erratum to: Theor Ecol (2015)

10.1007/s12080-015-0265-9

The original version of this article unfortunately contained a mistake. The correct name of "Ed McCauley" should be "Edward McCauley".

The online version of the original article can be found at http://dx.doi.org/ 10.1007/s12080-015-0265-9.

\section{Romain Richard}

romain.richard@univ-tours.fr

1 Department of Biological Sciences, University of Calgary,

Calgary, Alberta T2N 1N4, Canada

2 Institut de Recherche sur la Biologie de l'Insecte, IRBI UMR CNRS 7261, Université de Tours, 37200 Tours, France

3 Present address: Institut de Recherche sur la Biologie de l'Insecte, IRBI UMR CNRS 7261, Université de Tours, 37200 Tours, France 